\title{
Estimación por ondas ultrasónicas de la profundidad de la capa cementada en un acero $8620-\mathrm{H}^{(\bullet)}$
}

\author{
G.A. López-Huape ${ }^{(*)}$ y A. López-Ceja ${ }^{(*)}$ \\ Resumen Se llevó a cabo la estimación no destructiva de la capa cementada utilizando ondas ultrasónicas \\ Rayleigh en un acero 8620-H de bajo contenido de carbono, determinándose la relación entre la \\ amplitud de la onda dispersada Rayleigh y la profundidad de capa cementada. Estas investigaciones \\ parten del concepto de que la amplitud de la onda dispersada es atenuada por la microestructura de \\ acuerdo a una ley de mezclas entre el coeficiente de atenuación de la capa cementada y la no \\ cementada (material base), confirmándose con ello la investigación teórica. La amplitud de la onda \\ dispersada Rayleigh se medió en 4 tipos de probetas de diferentes espesores de acero cementado. \\ Estas mediciones indican que la amplitud de la onda dispersada decrece exponencialmente de \\ acuerdo con los espesores medidos de cementación, como una expresión contrastante a la \\ investigación teórica. \\ Palabras clave: Onda dispersada. Capa cementada. Onda Rayleigh. Amplitud. Espesores de \\ cementación.

\section{Use of ultrasonic waves for estimating the carburized case depth in a $8620-\mathrm{H}$ steel}

\begin{abstract}
Non destructive estimation of the carburize case depth by using ultrasonic Rayleigh waves in a 8620$\mathrm{H}$ low carbon steel. Before the experimental work the relationship between backscattered Rayleigh wave amplitud and carburized depth was investigated. These studies depend on the concept that the amplitud of backscattered waves is attenuated by the microstructure according to a combined law established between the attenuation coefficient of the carburized case and the noncarburized base material, confirming the results of the theoretical study. The amplitude of the backscattered Rayleigh wave was measured in four tests of different thicknesses of the carburized case. These measurements indicated that the amplitud of the wave decreases exponentially in accordance with the carburized case measured, as an expression which contracts with the theoretical investigation.
\end{abstract}

Keywords: Backscattered wave. Carburized case. Rayleigh wave. Amplitude. Thicknes of carburization.

\section{INTRODUCCIÓN}

La industria metal-mecánica requiere la estimación precisa y rápida de la profundidad de la capa cementada de aceros, principalmente para la optimización del proceso de control de calidad en línea,

(•) Trabajo recibido el día 11 de septiembre de 1995.

(*) Instituto de Investigaciones Metalúrgicas. Universidad Michoacana de San Nicolás de Hidalgo. Aptdo. Postal 52-B. Morelia, Mich. 58000 (México). ya que actualmente la determinación convencional se lleva a cabo mediante un ensayo destructivo de microdureza, que implica cierto consumo de tiempo. En este trabajo se intenta determinar la profundidad de la capa cementada mediante la utilización de ondas dispersadas Rayleigh, observándose su sensibilidad al paso por la profundidad de la capa cementada, por la concentración de su energía en ese volumen superior de la capa. Cuando se desplazan a lo largo de la probeta las ondas Rayleigh dispersadas, se atenúan por causa de las microestructuras de las dos capas, incrementándose el valor de la 
atenuación en relación con el espesor de capa cementada, por lo que se afirma que las propiedades acústicas de dicha capa y de la no cementada tienen diferentes coeficientes de dispersión. El presente trabajo se dividió en dos partes:

- Investigación teórica de la atenuación de las ondas dispersadas Rayleigh en el material cementado.

- Medida experimental de las ondas dispersadas Rayleigh en el material de prueba.

\section{INVESTIGACIÓN TEÓRICA}

Cuando una onda dispersada Rayleigh viaja a lo largo de la probeta de material cementado, como muestra la figura 1, la onda queda atenuada por dispersión microestructural. Esto ocurre si la frecuencia ultrasónica es baja y, por lo tanto, la longitud de onda es amplia, todo ello en relación con la profundidad de capa cementada, dando lugar a que la onda Rayleigh sea transmitida en ambas capas, cementada y no cementada. La amplitud de la onda dispersada Rayleigh depende de dos factores.

Uno de ellos es la distribución de la energía ultrasónica de las ondas en la dirección $z$ (Fig. 1), la cual queda atenuada exponencialmente de acuerdo con la profundidad de capa cementada. En ésta, la energía ultrasónica se expresa como una función de la forma siguiente [1] (1):

$$
\phi=\phi(\mu, \lambda, d)
$$

donde:

$\mu=$ Coeficiente de Poisson,

$\lambda=$ Longitud de onda,

$d=$ Profundidad de la capa cementada.

El otro factor es el coeficiente de atenuación, $\alpha$, del acero cementado. La amplitud de la onda dispersada total se puede expresar por [2] (1):

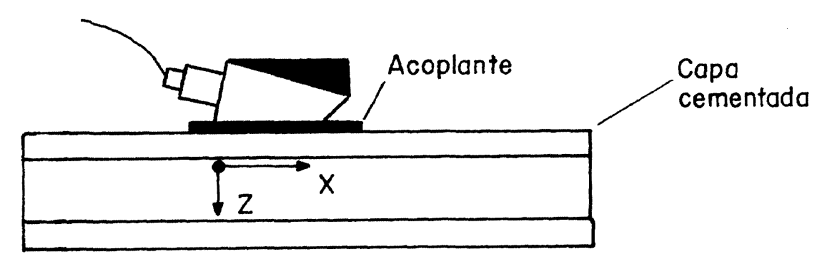

FIG. 1.- Propagación de una onda dispersada Rayleigh a lo largo de una probeta con las superficies cementadas.

FIG. 1.- Propagation of the ultrasonic Rayleigh in 8620-H steel carburized.

$$
H(d)=\frac{A_{\mathrm{I}}(x)}{A_{\mathrm{o}}}
$$

donde:

$A_{\mathrm{I}}(x)=$ Amplitud de la onda incidente,

$A_{\mathrm{o}} \quad=$ Amplitud de la onda dispersada Rayleigh,

$H(d)=$ Función de profundidad de capa cementada .

En el presente caso (el coeficiente de dispersión $\alpha_{d}$ causado por la dispersión es muy grande comparado con el coeficiente de absorción $\alpha_{\mathrm{a}}$, por lo que no se toma en cuenta), $\alpha=\alpha d$.

A partir de los factores anteriores, se propone la siguiente hipótesis: la atenuación resultante (en ambas fases) de las ondas dispersadas, es la superposición de las atenuaciones sufridas individualmente en cada una de las fases (capa cementada y material base), consideradas éstas como fases constituidas de material homogéneo. Además, se toma una frontera bien definida en el material. Esto se puede expresar de la siguiente manera:

$H(d)=$ Amplitud de la onda incidente/Amplitud de la onda dispersada

\section{ENSAYOS}

\subsection{Materiales de ensayo}

La composición química del acero de baja aleación, utilizado en el trabajo del análisis ultrasónico, es la que muestra la tabla I, de donde se deduce que dicho material (2) es un acero 8620-H (AISI). Las probetas se mecanizaron a las dimensiones que especifica la figura 2, preparándose con cuatro espesores diferentes de capa cementada y tratándose térmicamente según los valores que se ofrecen en la tabla II. Las figuras 3a) y 3b) muestran ejemplos de la microestructura de las probetas. En las figuras $4 a$ ) y 4 b) se observan los valores de dureza Vickers

TABLA I.- Resultados de la composición química

TABLE I.- Results of the chemical composition

\begin{tabular}{|c|c|c|}
\hline Elemento & Según (2), \% & Composición, \% \\
\hline $\mathrm{C}$ & $0,17-0,60$ & 0,187 \\
\hline $\mathrm{Mn}$ & $0,60-0,95$ & 0,908 \\
\hline $\mathrm{P}$ & 0,035 & 0,013 \\
\hline $\mathrm{S}$ & 0,40 máx. & 0,029 \\
\hline $\mathrm{Si}$ & $0,15-0,30$ & 0,233 \\
\hline $\mathrm{Ni}$ & $0,35-0,75$ & 0,482 \\
\hline $\mathrm{Cr}$ & $0,35-0,65$ & 0,436 \\
\hline $\mathrm{Mo}$ & $0,15-0,25$ & 0,206 \\
\hline
\end{tabular}


ACERO $8620-\mathrm{H}$

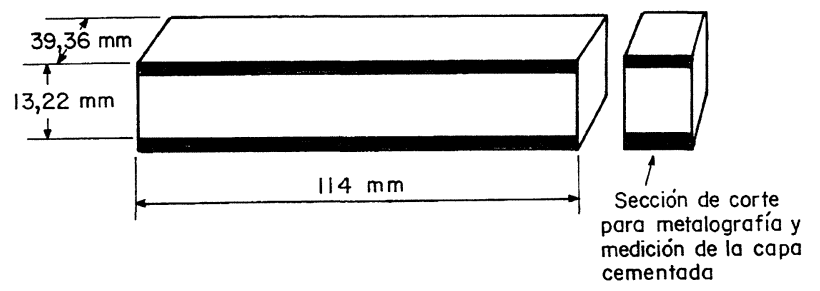

FIG. 2.- Dimensiones de las probetas de ensayo.

FIG. 2.-Dimensions of the specimen.

TABLA II.- Tratamientos térmicos

TABLE II.- Heat treatments

\begin{tabular}{|c|c|c|c|}
\hline Probeta & $\begin{array}{c}\text { Temperatura, } \\
{ }^{\circ} \mathrm{C}\end{array}$ & $\begin{array}{c}\text { Tiempo en el } \\
\text { horno, } \mathrm{h}\end{array}$ & $\begin{array}{c}\text { Profundidad } \\
\text { efectiva de } \\
\text { capa cemen- } \\
\text { tada, } \mathrm{mm}\end{array}$ \\
\hline P0 & 25 & Sin tratamiento & 0 \\
\hline P1 & 950 & 5 & 0,731 \\
\hline P2 & 950 & 8 & 1,392 \\
\hline P3 & 950 & 12 & 2,049 \\
\hline
\end{tabular}

distribuidos en el área subsuperficial para P1 y P3. En este trabajo, se considera que el valor de $500 \mathrm{HV}$ define el límite de la profundidad de capa efectiva basado en la norma ASM E384-84. Para los presentes ensayos, la profundidad de espesor de capa cementada efectiva varía de 0 a 2,049 $\mathrm{mm}$.

\section{INSTALACIÓN EXPERIMENTAL}

La instalación experimental del sistema de medición ultrasónico se muestra en la figura 5. Este sistema se caracteriza por tener una velocidad de procesamiento de $10 \mathrm{GSa} / \mathrm{s}$ y una frecuencia de trabajo máxima de $150 \mathrm{MHz}$, digitalizando además la señal, por lo que puede medir con precisión la amplitud de la onda dispersada Rayleigh. Este sistema ultrasónico comprende los siguientes equipos: uno de ultrasonidos marca Panametrics, modelo 5800, por medio del cual se realiza la excitación de la señal ultrasónica; un osciloscopio digital marca Hewlett Packard, modelo 54602A, para la medición de las características ultrasónicas de la señal de la onda dispersada Rayleigh; un ordenador marca Acer Power, modelo 486Dx, para el almacenamiento, estudio y procesamiento de los datos experimentales; un transductor de frecuencia central de $5 \mathrm{MHz}$, que es el que mejor se adaptó al ensayo de medición; una zapata de $90^{\circ}$ de punto focal, de resina acrílica, que se utilizó como acoplamiento entre el transductor y la pieza de

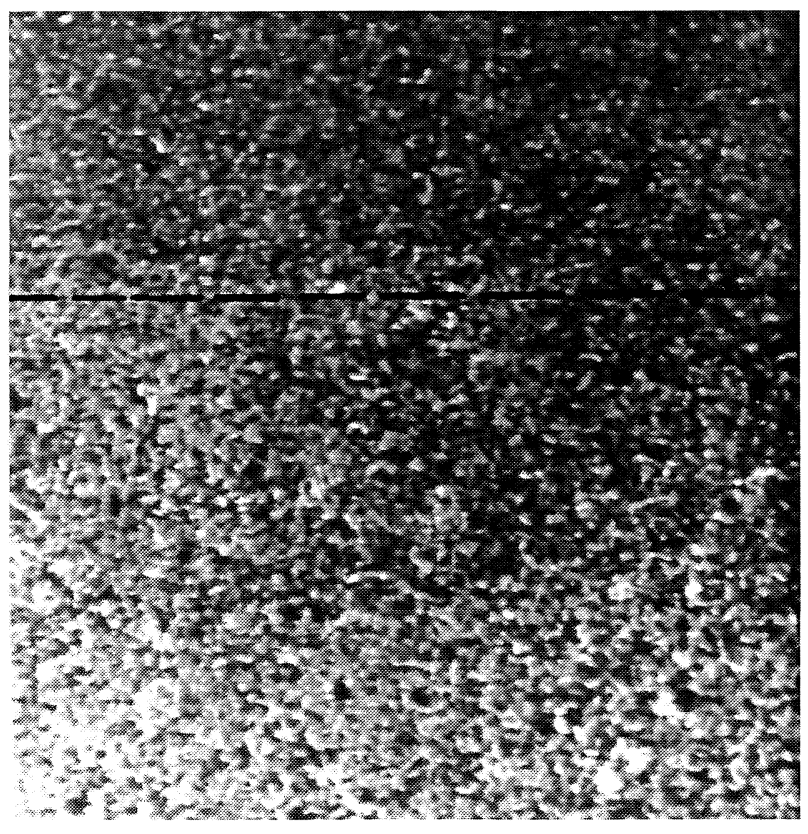

FIG. 3a).- Micrografía de la probeta P2, $950{ }^{\circ} \mathrm{C}$, 8 h. Línea divisoria entre capa cementada y no cementada. $\times 100$.

FIG. 3a).-Micrography specimen P2, $950{ }^{\circ} \mathrm{C}, 8 \mathrm{~h}$. Line divide between carburized case and uncarburized $\times 100$.

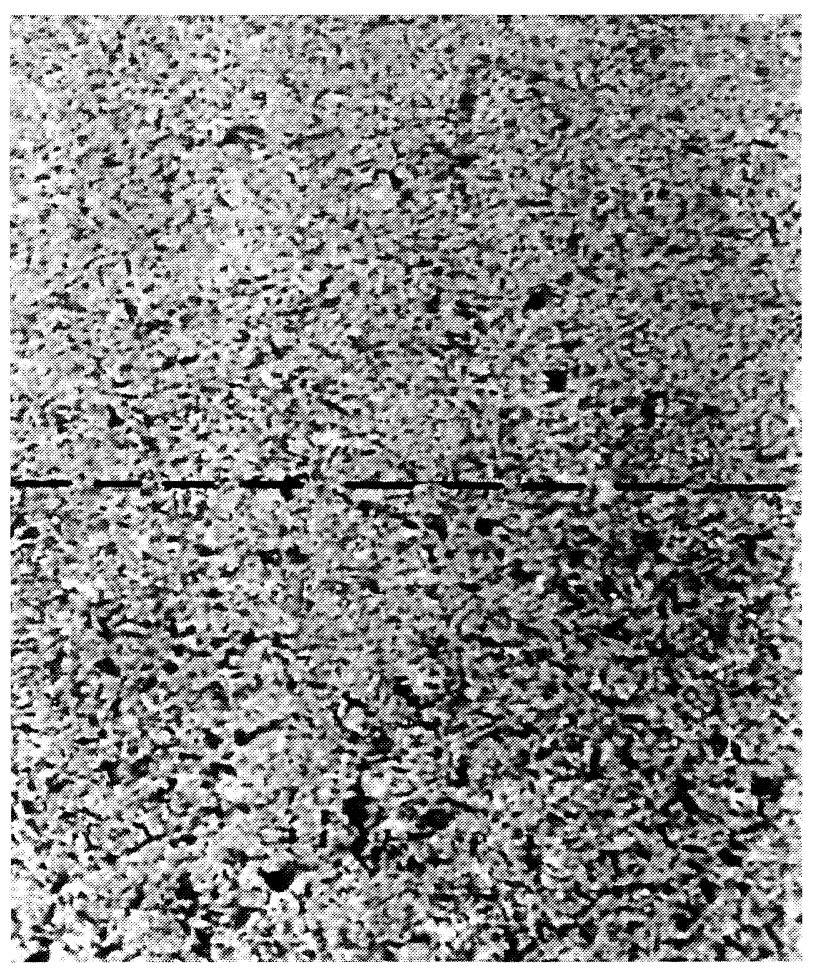

FIG. 3b).- Micrografía de la probeta $\mathrm{P} 3,950{ }^{\circ} \mathrm{C}$, 12 h. Línea divisoria entre capa cementada y no cementada. $\times 100$.

FIG. 3b).- Micrography specimen P3, $950{ }^{\circ} \mathrm{C}$, $12 \mathrm{~h}$. Line divide between carburized case and uncarburized $\times 100$. 


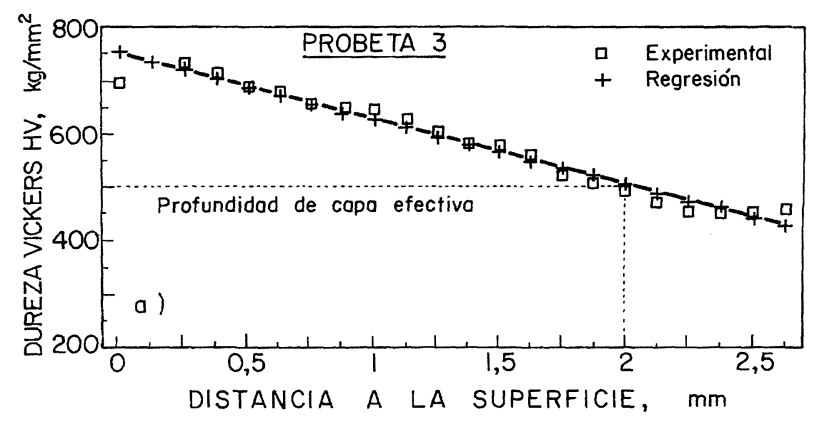

FIG. 4a).- Valores de microdureza de la capa cementada de la probeta $\mathrm{P} 3$.

FIG. 4a).- Microhardness values of the carburized case depth in specimen $P 3$.

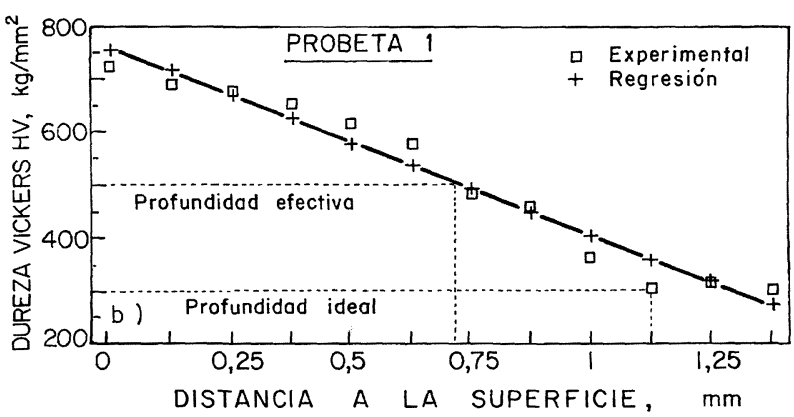

FIG. 4b).- Valores de microdureza de la capa cementada de la probeta $\mathrm{P} 1$.

FIG. 4b).- Microhardness values of the carburized case depth in specimen P1.

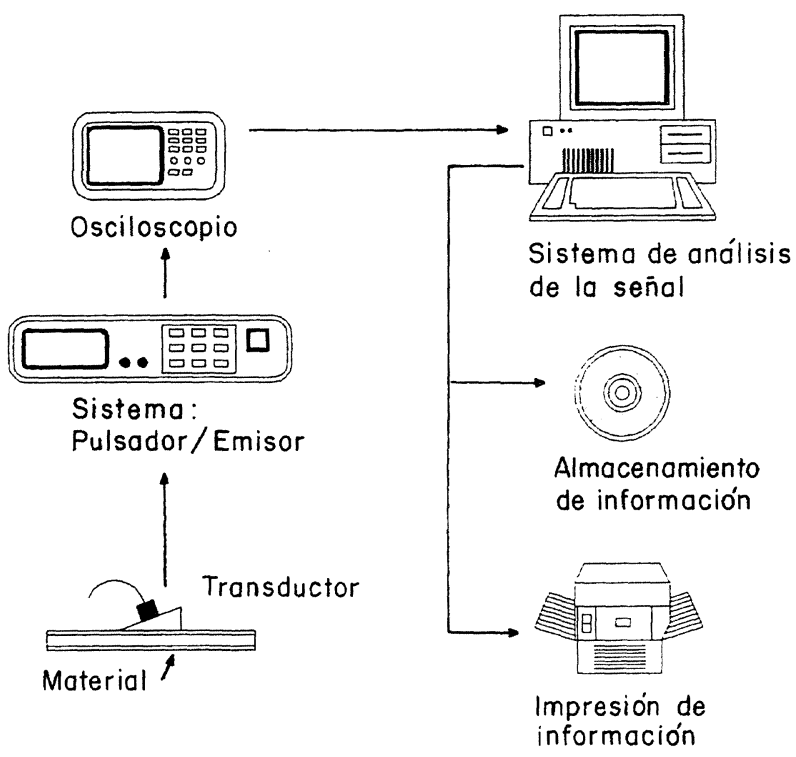

Fig. 5.- Representación esquemática de los equipos de ensayo.

FIG. 5.- Representation graphic of the equipment of test. trabajo (estas características proporcionan a la zapata la posibilidad de transmitir las ondas dispersadas debido a su bajo coeficiente de atenuación para transmitir las ondas Rayleigh (3). Dicha zapata tiene la misión de transmitir la onda Rayleigh y de recibir la onda dispersada.

\section{DESARROLLO EXPERIMENTAL}

Las probetas de acero a cementar se analizaron químicamente por espectrofotometría de absorción atómica y por infrarrojo (Tabla I). Acto seguido se cementaron en un horno en las condiciones de temperatura, presión y tiempo que indica la tabla II. Cabe mencionar que el proceso de cementación se rige por los mecanismos de absorción y de difusión de Fick (4).

Las probetas cementadas se mecanizaron a las medidas adecuadas al diseño del ensayo ultrasónico, cuyas restricciones fueron la rugosidad y el área de medición, resultando la geometría de las probetas que muestra la figura 2 , y se realizaron análisis metalográficos de las capas de acero cementado y no cementado, cuyos resultados muestran las micrografías de las figuras 3a) y 3b) (5).

Posteriormente, se realizó el estudio ultrasónico aplicando el método de impulso-eco, con la técnica de contacto. Se fijó el transductor en una placa acrílica, para mantener su rigidez y direccionar el haz sónico a la superficie de rastreo. Antes del posicionamiento del transductor, se colocó grasa automotriz como acoplante entre la placa de acrílico y el material a ensayar. En el desarrollo de la medición se observó en la pantalla del osciloscopio la señal tipo scan "A", que muestra la amplitud de la señal ultrasónica Rayleigh (Figs. 6a), 6b), 6c) y 6d). Por último, a partir de las señales en el dominio del tiempo, se les aplico la FFT (Transformada rápida de Fourier) que calcula las componentes de frecuencia que muestran las figuras $6 a$ ), 6b), 6c) y 6d) y la figura 7. A continuación, se analizaron las señales obtenidas, complementándose con un análisis estadístico y correlacionando las variables de estudio (la amplitud de la onda dispersada, el espesor de capa cementada y la microdureza obtenida) obteniéndose los datos que muestran las figuras 4a) y $4 b)$.

\section{RESULTADOS EXPERIMENTALES}

La tabla I muestra la composición química del acero 8620-H (AISI) utilizado en este estudio, y la tabla II los valores de profundidad efectiva de la capa cementada para cada una de las probetas obtenidas experimentalmente y los valores del tratamiento térmico a que se sometieron. Las figuras 3a) 


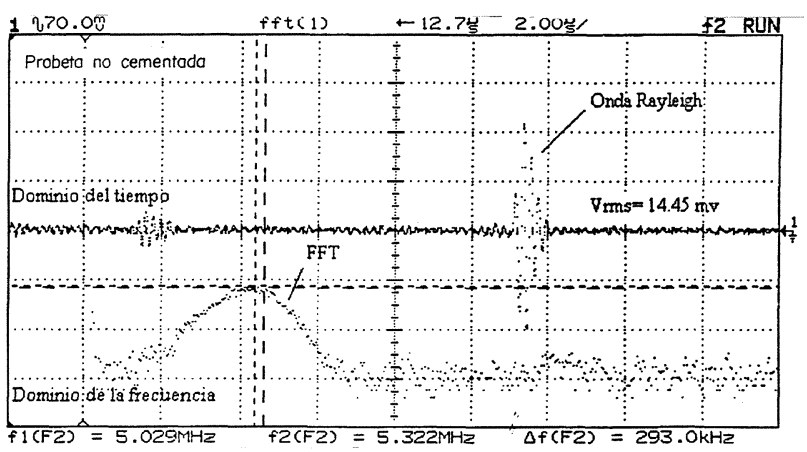

FIG. 6a).- Amplitud de la onda ultrasónica Rayleigh; probeta no cementada.

FIG. 6a).- Amplitude of the ultrasonic Rayleigh waves in specimen uncarburized.

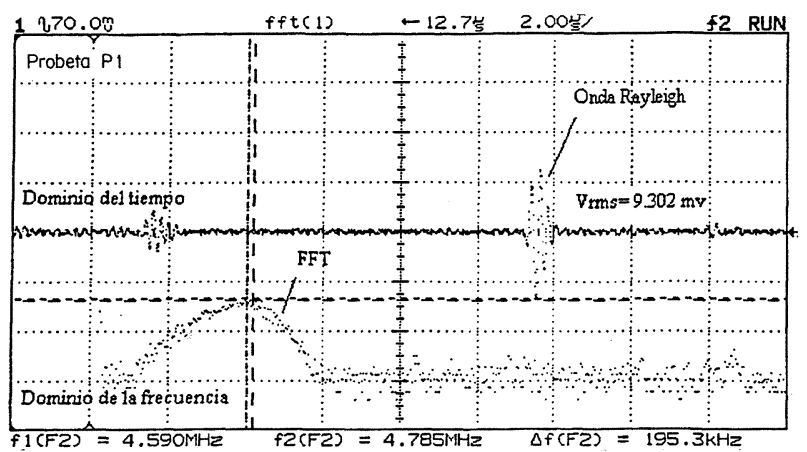

FIG. 6b).- Amplitud de la onda ultrasónica Rayleigh en la probeta cementada P1.

FIG. 6b).- Amplitude of the ultrasonic Rayleigh waves in specimen carburized $P 1$.

y 3b) muestran las microestructuras de la capa cementada y de la no cementada de las probetas $\mathrm{P} 2$ y P3, y las figuras $4 a$ ) y 4 b) los valores de microdureza de la capa cementada en relación con el espesor. Las señales dispersadas para cada una de las probetas se muestran en las figuras $6 a), 6 b), 6 c)$ y $6 d)$, en las que cada señal corresponde a un reflector de referencia frente a una distancia específica de $14,36 \mathrm{~mm}$; esta reflexión se analiza en una ventana de $-12,7$ ns a 2,00 ns. En la figura 6, para análisis de las señales dispersadas, la tensión efectiva se ajustó a un simple parámetro. La figura 8 muestra la relación entre la onda dispersada y la profundidad de capa para cada probeta. La línea punteada indica la correlación matemática de forma exponencial con un factor de correlación del $98 \%$ estadísticamente. La figura 7 muestra la relación que guarda la frecuencia con respecto al espesor de la capa cementada, observándose que disminuye la frecuencia conforme aumenta el valor del espesor de la capa. El comportamiento del parámetro ultrasónico de frecuencia con la capa cementada se muestra en las figuras 6a), 6b), 6c) y 6d).

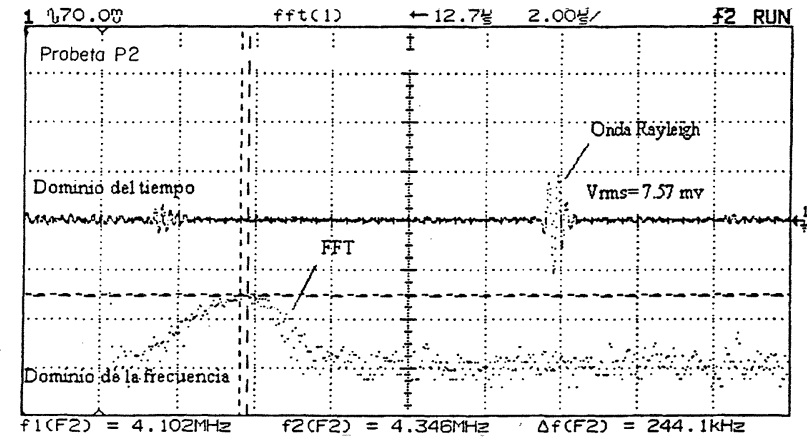

FIG. 6c).- Amplitud de la onda ultrasónica Rayleigh en la probeta cementada $\mathrm{P} 2$.

FIG. 6c).- Amplitude of the ultrasonic Rayleigh waves in specimen carburized $P 2$.

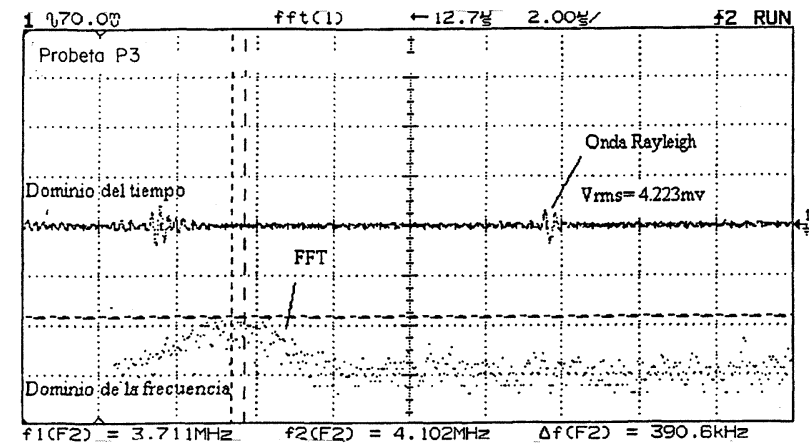

FIG. 6d).- Amplitud de la onda ultrasónica Rayleigh en la probeta cementada P3.

FIG. 6d).-Amplitude of the ultrasonic Rayleigh waves in specimen carburized P3.

\section{DISCUSION}

En el presente estudio se investigan las propiedades ultrasónicas de aceros cementados para la estimación del espesor de la capa cementada por medio de la utilización de ondas dispersadas

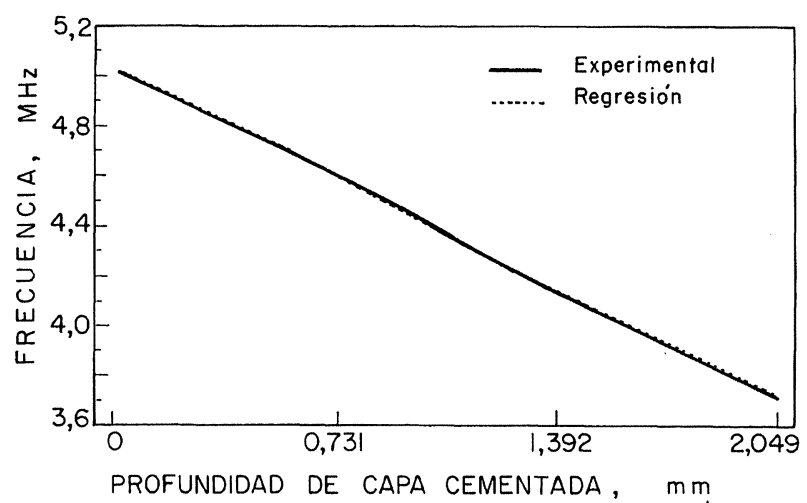

FIG. 7.- Amplitud de la señal con respecto al espesor de la capa cementada.

FIG. 7.- Amplitude of the sign with respect the thicknes of the carburized. 
Rayleigh. La transmisión y la dispersión de la onda ultrasónica Rayleigh se explican cuantitativamente con un simple modelo de ley de mezclas, que cumple la ecuación [2] que contiene algunas restricciones especiales, como es la de considerar que la amplitud de la onda dispersada se incrementa exponencialmente de acuerdo con el espesor de la capa cementada, donde el coeficiente de dispersión es grande comparado con el coeficiente de absorción. Experimentalmente, se observó que es muy importante una buena selección de la frecuencia de trabajo, ya que a frecuencias elevadas se tiene una atenuación alta, que imposibilita la medida de la amplitud de esa señal; por el contrario, a frecuencias muy bajas se consigue una elevada penetración de la onda en el material, que da como resultado la pérdida de sensibilidad en la capa cementada, por lo que no se puede medir la amplitud de la señal en estudio. La secuencia de las figuras 6a) a 6d) muestra el decremento de la amplitud dispersada, observándose que existe una relación con el incremento de espesor de capa cementada, prediciendo que la onda dispersada puede ser aplicable para la estimación de dicha capa. La figura 8 es la representación gráfica más importante, ya que muestra la relación que existe entre la amplitud de la onda dispersada Rayleigh con el incremento de la capa cementada, siendo esta relación inversamente exponencial.

La figura 7 muestra el comportamiento de la frecuencia, observándose que este parámetro ultrasónico es más sensible a la interacción de la capa cementada que con respecto a la amplitud de la

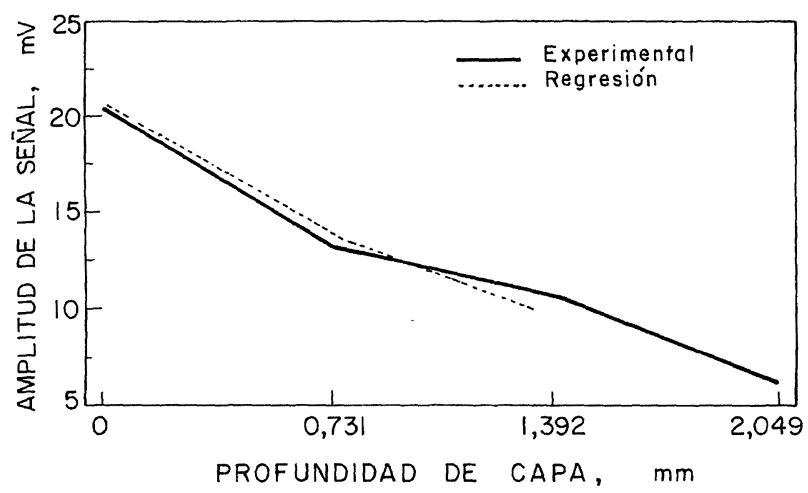

FIG. 8).- Valores de frecuencia frente al espesor de la capa cementada.

FIG. 8).- Value of frequency with respect the thicknes of the carburize case depth. señal. La figura 5 muestra esquemáticamente la instalación experimental, que resultó adecuada para el estudio de la estimación de la profundidad de la capa cementada.

\section{CONCLUSIONES}

- De los resultados presentados en las figuras 6a), 6b), 6c) y 6d), se concluye que existe buena relación entre el espesor de capa cementada y la amplitud de la onda dispersada Rayleigh, sugerida por un modelo teórico basado en una simple ley de mezclas.

- Las figuras 4a) y 4b) determinan que existe buena relación entre la microdureza con el espesor de la capa cementada, contrastando con la amplitud de la onda dispersada Rayleigh.

- De la figura 7 se deduce que en la frecuencia influye más la interacción con la capa cementa$\mathrm{da}$, con la que tiene un comportamiento inverso.

- En la aplicación de este modelo, para la medición ultrasónica de capas endurecidas, es muy importante una selección adecuada de la frecuencia de trabajo para cada caso en particular.

- El modelo de mezclas podría estudiarse para probables aplicaciones en otros tipos de superficies endurecidas.

- La instalación experimental ultrasónica adaptada al proyecto de investigación de estimación de capa cementada fue la adecuada para el logro de los resultados mostrados y su potencialidad podría utilizarse en otros estudios de caracterización no destructiva de materiales.

\section{REFERENCIAS}

(1) Mihara, T. у Obata, M. Mater. Eval., 49 (6), 1991: 696700.

(2) Pascual, J. Técnica y Práctica del Tratamiento Térmico de Los Metales Férreos. Ed. Blume. Barcelona (España), 1970: 449.

(3) Davis, J.R. Metals Handbook. Ninth Edition. Vol. 17. Nondestructive Evaluation and Quality Control. Ed. ASM, (EE.UU.), 1990: 235.

(4) Krauss, G. Carburizing Processing and Performance. Ed. ASM, (EE.UU.), 1989: 41-56.

(5) Parrish, G. The Influencie of Microstructure on the Properties of Case-carburized Components. Ed. ASM, (EE.UU.), 1980: 23-38. 